\title{
Opacity in compact extragalactic radio sources and its effect on radio-optical reference frame alignment
}

\author{
Y. Y. Kovalev ${ }^{1,2}$, A. P. Lobanov ${ }^{1}$, A. B. Pushkarev ${ }^{1,3,4}$, J. A. Zensus ${ }^{1}$ \\ ${ }^{1}$ Max-Planck-Institut für Radioastronomie, Auf dem Hügel 69, 53121 Bonn, Germany \\ e-mail: ykovalev, alobanov, apushkar, azensus@mpifr-bonn.mpg.de \\ ${ }^{2}$ Astro Space Center of Lebedev Physical Institute, \\ Profsoyuznaya 84/32, 117997 Moscow, Russia \\ ${ }^{3}$ Pulkovo Astronomical Observatory, Russia; ${ }^{4}$ Crimean Astrophysical Observatory, Ukraine
}

\begin{abstract}
Accurate alignment of the radio and optical celestial reference frames requires detailed understanding of physical factors that may cause offsets between the positions of the same object measured in different spectral bands. Opacity in compact extragalactic jets (due to synchrotron self-absorption and external free-free absorption) is one of the key physical phenomena producing such an offset, and this effect is well-known in radio astronomy ("core shift"). We have measured the core shifts in a sample of 29 bright compact extragalactic radio sources observed by Very Long Baseline Interferometry (VLBI) at 2.3 and $8.6 \mathrm{GHz}$. We report the results of these measurements and estimate that the average shift between radio and optical positions of distant quasars could be of the order of 0.1-0.2 mas. This shift exceeds the expected positional accuracy of Gaia and SIM. We suggest two possible approaches to carefully investigate and correct for this effect in order to align accurately the radio and optical positions. Both approaches involve determining a Primary Reference Sample of objects to be used for tying the radio and optical reference frames together.
\end{abstract}

Keywords. galaxies: active, galaxies: jets, radio continuum: galaxies, astrometry, reference systems

\section{Introduction}

Extragalactic relativistic jets are formed in the immediate vicinity of the central black holes in galaxies, at distances of the order of 100 gravitational radii, and they become visible in the radio at distances of about 1000 gravitational radii (Lobanov \& Zensus 2007). This apparent origin of the radio jets is commonly called the "core". In radio images of extragalactic jets, the core is located in the region with an optical depth $\tau_{s} \approx 1$. This causes the absolute position of the core, $r_{\text {core }}$, to vary with the observing frequency, $\nu$, since the optical depth profile along the jet depends on $\nu: r_{\text {core }} \propto \nu^{-1 / k_{\mathrm{r}}}$ (Blandford \& Königl 1979). Variations of the optical depth along the jet can result from synchrotron self-absorption (Königl 1981), pressure and density gradients in the jet and free-free absorption in the ambient medium most likely associated with the broad-line region (BLR) (Lobanov 1998).

The core shift is expected to introduce systematic offsets between the radio and optical positions of reference sources, affecting strongly the accuracy of the radio-optical matching of the astrometric catalogues. The magnitude of the core shift can exceed the inflated errors of the radio and optical positional measurements by a large factor. This makes it necessary to perform systematic studies of the core shift in the astrometric samples in 
order to understand and remove the contribution of the core shift to the errors of the radio-optical position alignment.

Measurements of the core shift have been done so far only in a small number of objects (e.g., Marcaide et al. 1994; Lara et al. 1994; Porcas \& Rioja 1997; Lobanov 1996, 1998; Ros \& Lobanov 2001; Kadler et al. 2004; Sokolov \& Cawthorne 2007). In this paper, we present results for 29 compact extragalactic radio sources used in VLBI astrometric studies and discuss the core shift effect on the alignment of radio and optical reference frames.

\section{Core shift measurements between 2.3 and $8.6 \mathrm{GHz}$}

We have imaged and analyzed 277 sources from ten $24 \mathrm{hr}$-long geodetic RDV (Research and Development VLBI experiments, see, e.g., Gordon 2005) observations obtained in 2002 and 2003. Geodetic RDV sessions feature simultaneous observations at $2.3 \mathrm{GHz}$ and 8.6 GHz (S and X bands) with a global VLBI network which for each session includes the VLBA and up to nine other radio telescopes around the world.

This long-term RDV program is one of the best opportunities for a large project to measure two-frequency core shifts for several reasons: (i) it is optimized to have a good $(u, v)$-coverage, (ii) it has the maximum possible resolution for ground-based VLBI at these frequencies, (iii) the frequency ratio between the simultaneously observed bands is high (3.7), and (iv) the core shift per unit of frequency between 2.3 and $8.6 \mathrm{GHz}$ is larger than that at higher frequencies (see, e.g., Lobanov 1998). A dedicated multi-band VLBI project covering a wider frequency range would certainly provide results of a better quality, but it is extremely time consuming.

We have measured the frequency-dependent core shift between 2.3 and $8.6 \mathrm{GHz}$ by model-fitting the source structure with two-dimensional Gaussian components (Pearson 1999) and referencing the position of the core component to one or more jet features, assuming the latter to be optically thin and having frequency-independent peak positions.

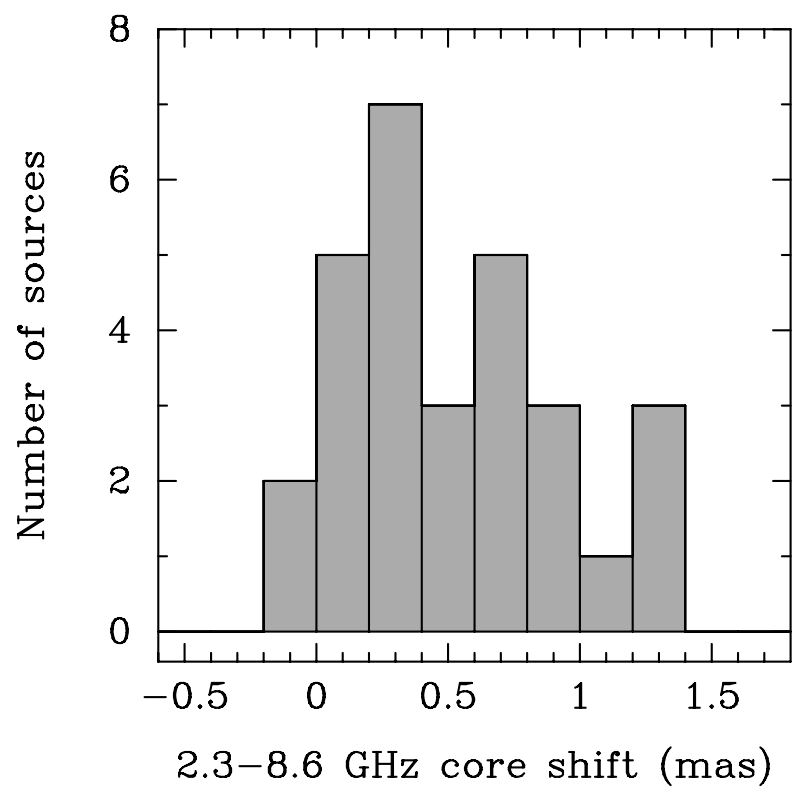

Figure 1. Histogram of the derived core shift values for 29 sources. One average core shift value per source is used. The median value for the distribution is equal to 0.44 mas. 
The shifts were successfully measured in 29 Active Galactic Nuclei (AGN), with the values of a shift ranging between -0.1 and 1.4 mas and the median value for the sample of 0.44 mas (Fig. 1). In this study, typical errors of the core shift measurements are about or less than 0.1 mas. For $90 \%$ of the 277 objects imaged, no reliable estimates of core shifts have been obtained by this method.

\section{Radio-optical alignment of astrometric positions}

The core shift issue gains specific importance when the radio reference frame needs to be linked to an optical reference frame. So far the link is based on the study of some radio stars which are seen both by Hipparcos and VLBI. These measurements are based on stars with large radiospheres and with big spots at their optical surfaces; both effects may introduce large uncertainties (see, e.g., Lestrade et al. 1995; Boboltz 2005; Ros 2005). Future accurate alignment of the frames has to rely on compact radio sources such as distant quasars.

Below, we discuss the alignment problem for compact extragalactic radio sources. We assume that the dominating component in both the radio and optical bands is the synchrotron self-absorbed compact jet origin (core). Broad-band modeling of blazar spectral energy distribution supports this hypothesis (see, e.g., recent review by Böttcher 2007). High-resolution VLBI observations of nearby AGN imply that the jet is formed and emitting in the radio already at distances of $\leqslant 1000$ gravitational radii from the central engine (e.g. Junor et al. 1999; Kadler et al. 2004). Thus the physical offset between the jet base and the central nucleus can be much smaller than the positional shift due to opacity in the jet (the latter can be larger than 1 pc). This implies that the offset between radio and optical positions of reference quasars will be dominated by the core shift even if the optical emission comes from the accretion disk around the central nucleus.

The magnitude of the core shift, $\Delta r$, between two arbitrary frequencies $\nu_{1}$ and $\nu_{2}$ $\left(\nu_{1}>\nu_{2}\right)$ caused by synchrotron self-absorption can be predicted for an object with known synchrotron luminosity, $L_{\text {syn }}$, of a compact jet (Lobanov 1998). If not corrected for, the core shift will introduce an additional error factor in the alignment of radio and optical reference frames. For typical parameters of relativistic jets we estimate the average shift between $8.6 \mathrm{GHz}$ and $6000 \AA$ for a complete sample of compact extragalactic sources to be on the order of 0.1-0.2 mas.

\section{Summary}

Measurements of frequency-dependent shifts in the parsec-scale jet cores in AGN are reported for 29 bright extragalactic radio sources. It is shown that the shift can be as high as 1.4 mas between 2.3 and $8.6 \mathrm{GHz}$. We have shown that the core shifts are likely to pose problems for linking radio and optical reference frames. We have estimated theoretically the average shift between the radio $(4 \mathrm{~cm}$ ) and optical (at $6000 \AA$ ) bands to be of an order of 0.1 mas for a complete sample of radio selected AGN.

The estimated radio-optical core shift exceeds the positional accuracy of Gaia and SIM. It implies that the core shift effect should be carefully investigated, and corrected for, in order to align accurately the radio and optical positions. Based on our study, we suggest two possible approaches, both involving the Primary Reference Sample of objects to be used for tying the radio and optical reference frames: 1) Firstly, multi-frequency VLBI measurements can be used for calculating the projected optical positions, assuming that the radio and optical emission regions are both dominated by a spatially compact component marginally resolved with VLBI and SIM and are point-like for Gaia. The 
discrepancies between the measured optical and radio positions can then be corrected for the predicted shifts, and the subsequent alignment of the radio and optical reference frames can be done using standard procedures. 2) Secondly, more conservative approach may also be applied, by employing the VLBI observations to identify and include in the Primary Reference Sample only those quasars in which no significant core shift has been detected in multi-epoch experiments. Either of the two approaches should lead to a substantial improvement of the accuracy of radio-optical positional alignment.

\section{Acknowledgements}

These proceedings are based on a paper by Y. Y. Kovalev et al. (A\&A, 2007, submitted). The raw VLBI data were provided to us from the open NRAO archive. The National Radio Astronomy Observatory is a facility of the National Science Foundation operated under cooperative agreement by Associated Universities, Inc. Y. Y. Kovalev is a Research Fellow of the Alexander von Humboldt Foundation. Y. Y. Kovalev was supported in part by the Russian Foundation for Basic Research (project 05-02-17377) while working in Moscow in the first half of 2006. We would like to thank Patrick Charlot, Ed Fomalont, Leonid Petrov, Richard Porcas, Eduardo Ros as well as the NASA GSFC VLBI group and the MOJAVE team for fruitful discussions.

\section{References}

Blandford, R. D. \& Königl, A. 1979, ApJ, 232, 34

Boboltz, D. A. 2005, in: ASP Conf. Ser. 340, Future Directions in High Resolution Astronomy, J. Romney \& M. Reid (eds.), p. 342

Böttcher, M. 2007, Ap\&SSS, 309, 95

Gordon, D. 2005, in: ASP Conf. Ser. 340, Future Directions in High Resolution Astronomy J. Romney \& M. Reid (eds.), p. 496

Junor, W., Biretta, J. A., \& Livio, M. 1999, Nature, 401, 891

Kadler, M., Ros, E., Lobanov, A. P., Falcke, H., \& Zensus, J. A. 2004, A\&̊A, 426, 481

Königl, A. 1981, ApJ, 243, 700

Lara, L., Alberdi, A., Marcaide, J. M., \& Muxlow, T. W. B. 1994, A\&\&A, 285, 393

Lestrade, J.-F., et al. 1995, A\&SA, 304, 182

Lobanov, A. \& Zensus, J. A. 2007, in: Exploring the Cosmic Frontier, ESO Astrophysics Symposia, A. P. Lobanov, J. A. Zensus, C. Cesarsky, \& P. J. Diamond (eds.), p. 147

Lobanov, A. P. 1996, PhD thesis, New Mexico Institute of Mining and Technology, Socorro, NM, USA (1996)

Lobanov, A. P. 1998, A\&A, 330, 79

Marcaide, J. M., Elosegui, P., \& Shapiro, I. I. 1994, AJ, 108, 368

Pearson, T. J. 1999, in: ASP Conf. Ser. 180, Synthesis Imaging in Radio Astronomy II, G. B. Taylor, C. L. Carilli, \& R. A. Perley (eds.), p. 335

Porcas, R. W. \& Rioja, M. J. 1997, in: Proceedings of the 12th working meeting on European VLBI for Geodesy and Astrometry, B. R. Pettersen (ed.), p. 133

Ros, E. 2005, in: ASP Conf. Ser. 340, Future Directions in High Resolution Astronomy, J. Romney \& M. Reid (eds.), p. 482

Ros, E. \& Lobanov, A. P. 2001, in: Proceedings of the 15th Workshop Meeting on European VLBI for Geodesy and Astrometry, D. Behrend \& A. Rius (eds.), p. 208

Sokolov, A. \& Cawthorne, T. V. 2007, in: ASP Conf. Ser., Extragalactic Jets: Theory and Observations from Radio to Gamma Rays, T. A. Rector \& D. S. De Young (eds.), in press 